\title{
Influence of Calcium Scaling on Corrosion Behavior of Steel and
}

\section{Aluminum Alloys}

Gandhi R. Osorio-Celestinot, Miguel Hernandez ${ }^{\ddagger}$, Diego SolisIbarras, Samuel Tehuacanero-Cuapa, Arturo Rodríguez-Gómez, A. Paulina Gómora-Figueroa ${ }^{+*}$

† División de Ingeniería en Ciencias de la Tierra, Facultad de Ingeniería, Universidad Nacional Autónoma de México, Circuito Exterior, Ciudad Universitaria, 04510, Ciudad de México, Mexico

F Centro de Ingeniería de Superficies y Acabados (CENISA), Depto. de Materiales y Manufactura, Facultad de Ingeniería, UNAM, 04510, Ciudad de México, Mexico.

\$ Instituto de Investigaciones en Materiales, UNAM, Circuito Exterior, Ciudad Universitaria, 04510, Ciudad de México, Mexico

Instituto de Física, UNAM, Circuito Exterior, Ciudad Universitaria, 04510, Ciudad de México, Mexico

*Corresponding Author: p.gomora@unam.mx 


\section{Supporting Information}

\section{INDEX}

\section{TABLES}

Table S1. EDS data for Steel 1045_A and Steel 1045_B

Table S2. EDS data for Steel 304-2B_A and Steel 304-2B_B

Table S3. EDS data for AA 2024-T3_A and AA 2024-T3_B

Table S4. EDS data for AA 3003-H14

Table S5. Mechanical profilometry results before immersion in scaling environments (Brines 2-4).

Table S6. Coupons exposed area and scale mass for environment saturated with Brines 2, 3, and 4.

\section{FIGURES}

Figure S1. Scanning Electron Microscopy and composition analysis for Steel 1045 before corrosion test.

Figure S2. Scanning Electron Microscopy and composition analysis for Steel 304-2B before corrosion test.

Figure S3. Scanning Electron Microscopy and composition analysis for AA 2024-T3 before corrosion test.

Figure S4. Scanning Electron Microscopy and composition analysis for AA 3003-H14 before corrosion test.

Figure S5. PXRD of scales growth from brine 2, 3, and 4 for 304-2B and 3003-H14 coupons.

Figure S6. PXRD of scales growth from brine 2, 3, and 4 for 1045 and 2024-T3 coupons.

Figure S7. Mechanical profilometry diagrams for AA 3003-H14 and Steel 304-2B before and after grounding. 
Figure S8. Mechanical profilometry diagrams for AA 2024-T3 and Steel 1045 before and after grounding.

Figure S9. Backscattered electron micrographs for corrosive and scaling environments; A: 1045, C: 304-2B, E: 2024-T3, G: 3003-H14 and secondary electron micrographs for B: 1045, D: 304-2B, F: 2024-T3, H: 3003-H14.

Figure S10. Backscattered electron micrographs for base line experiments, non-scaling; A: 304-2B, C: 2024-T3, E: 3003-H14 and secondary electron micrographs for B: 304-2B, D: 2024-T3, F: 3003H14.

Figure S11. EIS data for 304-2B and 1045 at $0 \mathrm{~h}$ and $576 \mathrm{~h}$ of testing: Nyquist (A) and Bode (B)

Figure S12. EIS data for 3003-H14 and 2024-T3 at $0 \mathrm{~h}$ and $576 \mathrm{~h}$ of testing: Nyquist (A) and Bode (B)

Figure S13. EIS data for base line experiments (non-scaling environment, Brine 1): Nyquist (A) and Bode (B)

Figure S14. Electrical capacitance and resistance of the bare steel and aluminum alloys for the base line experiments (non-scaling conditions).
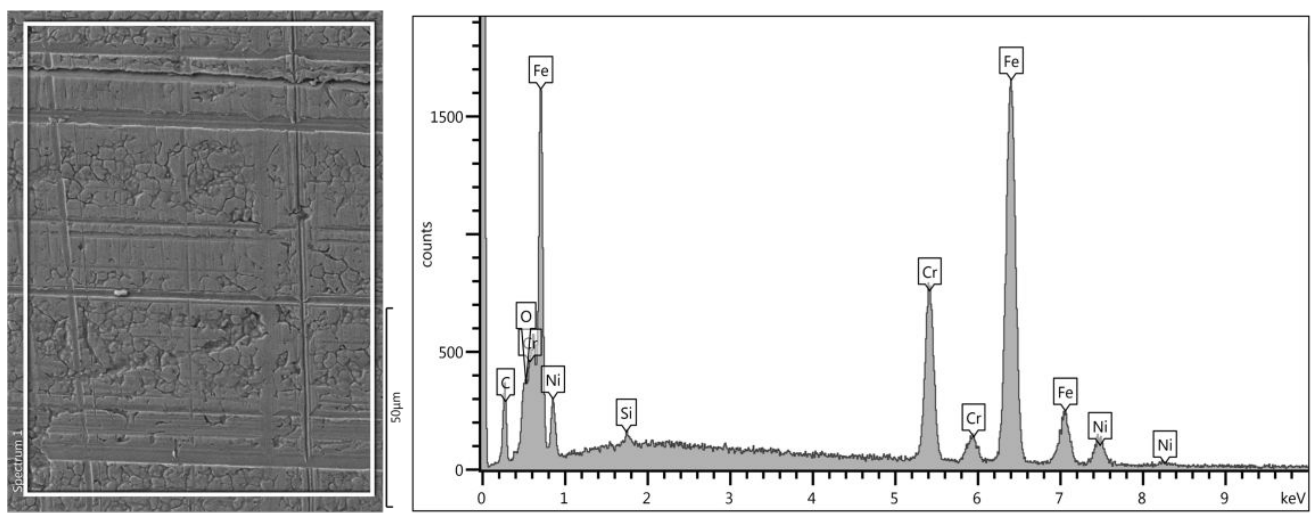

Figure S1. Scanning Electron Microscopy and composition analysis for Steel 1045

Table S1. EDS data for Steel 1045_A and Steel 1045_B.

\begin{tabular}{|c|c|c|c|c|c|c|c|c|}
\hline $\begin{array}{c}\text { Elemen } \\
t\end{array}$ & $\begin{array}{c}\text { Line } \\
\text { Type }\end{array}$ & $\begin{array}{c}\text { Apparent } \\
\text { Concentratio } \\
\mathrm{n}\end{array}$ & $\begin{array}{c}\mathrm{k} \\
\text { Ratio }\end{array}$ & wt\% & $\begin{array}{c}\text { wt } \\
\text { Sigm } \\
\mathrm{a}\end{array}$ & $\begin{array}{c}\text { Atomi } \\
\mathrm{C} \%\end{array}$ & $\begin{array}{c}\text { Standar } \\
\mathrm{d} \text { Label }\end{array}$ & $\begin{array}{c}\text { Factory } \\
\text { Standar } \\
\mathrm{d}\end{array}$ \\
\hline $\mathrm{C}$ & $\begin{array}{c}\mathrm{K} \\
\text { serie } \\
\mathrm{s}\end{array}$ & 0.57 & $\begin{array}{c}0.0057 \\
4\end{array}$ & 12.24 & 0.44 & 37.76 & C Vit & Yes \\
\hline
\end{tabular}




\begin{tabular}{|c|c|c|c|c|c|c|c|c|}
\hline 0 & $\begin{array}{c}\text { K } \\
\text { serie } \\
\text { s }\end{array}$ & 0.52 & $\begin{array}{c}0.0017 \\
4\end{array}$ & 1.90 & 0.17 & 4.39 & SiO2 & Yes \\
\hline $\mathrm{Si}$ & $\begin{array}{c}\text { K } \\
\text { serie } \\
\text { s }\end{array}$ & 0.07 & $\begin{array}{c}0.0005 \\
6\end{array}$ & 0.47 & 0.07 & 0.62 & $\mathrm{SiO} 2$ & Yes \\
\hline $\mathrm{Cr}$ & $\begin{array}{c}\text { K } \\
\text { serie } \\
\text { s } \\
\end{array}$ & 3.21 & $\begin{array}{c}0.0321 \\
4\end{array}$ & 15.92 & 0.28 & 11.35 & $\mathrm{Cr}$ & Yes \\
\hline $\mathrm{Fe}$ & $\begin{array}{c}\text { K } \\
\text { serie } \\
\text { s }\end{array}$ & 11.15 & $\begin{array}{c}0.1114 \\
6\end{array}$ & 62.66 & 0.51 & 41.58 & $\mathrm{Fe}$ & Yes \\
\hline $\mathrm{Ni}$ & $\begin{array}{c}K \\
\text { serie } \\
\text { s }\end{array}$ & 1.15 & $\begin{array}{c}0.0115 \\
5\end{array}$ & 6.81 & 0.37 & 4.30 & $\mathrm{Ni}$ & Yes \\
\hline Total: & & & & $\begin{array}{c}100.0 \\
0\end{array}$ & & $\begin{array}{c}100.0 \\
0\end{array}$ & & \\
\hline
\end{tabular}

\begin{tabular}{|c|c|c|c|c|c|c|c|c|}
\hline $\begin{array}{c}\text { Elemen } \\
t\end{array}$ & $\begin{array}{l}\text { Line } \\
\text { Type }\end{array}$ & $\begin{array}{c}\text { Apparent } \\
\text { Concentratio } \\
n\end{array}$ & $\begin{array}{c}\text { k } \\
\text { Ratio }\end{array}$ & wt $\%$ & $\begin{array}{l}\text { wt\% } \\
\text { Sigm } \\
\text { a }\end{array}$ & $\begin{array}{c}\text { Atomi } \\
\text { C } \frac{\circ}{0}\end{array}$ & $\begin{array}{l}\text { Standar } \\
\text { d Label }\end{array}$ & $\begin{array}{c}\text { Factory } \\
\text { Standar } \\
\text { d }\end{array}$ \\
\hline C & $\begin{array}{c}\text { K } \\
\text { serie } \\
\text { s }\end{array}$ & 0.36 & $\begin{array}{c}0.0036 \\
5\end{array}$ & 8.41 & 0.42 & 29.22 & C Vit & Yes \\
\hline 0 & $\begin{array}{c}\text { K } \\
\text { serie } \\
\text { s }\end{array}$ & 0.23 & $\begin{array}{c}0.0007 \\
7\end{array}$ & 0.82 & 0.16 & 2.13 & SiO2 & Yes \\
\hline $\mathrm{Si}$ & $\begin{array}{c}\text { K } \\
\text { serie } \\
\text { s }\end{array}$ & 0.04 & $\begin{array}{c}0.0002 \\
8\end{array}$ & 0.25 & 0.07 & 0.38 & $\mathrm{SiO} 2$ & Yes \\
\hline $\mathrm{Cr}$ & $\begin{array}{c}\mathrm{K} \\
\text { serie } \\
\mathrm{s} \\
\end{array}$ & 3.28 & $\begin{array}{c}0.0328 \\
4\end{array}$ & 16.78 & 0.29 & 13.46 & $\mathrm{Cr}$ & Yes \\
\hline $\mathrm{Fe}$ & $\begin{array}{c}\text { K } \\
\text { serie } \\
\text { s }\end{array}$ & 11.38 & $\begin{array}{c}0.1138 \\
1\end{array}$ & 66.05 & 0.52 & 49.34 & $\mathrm{Fe}$ & Yes \\
\hline $\mathrm{Ni}$ & $\begin{array}{c}K \\
\text { serie } \\
\text { s }\end{array}$ & 1.26 & $\begin{array}{c}0.0125 \\
8\end{array}$ & 7.69 & 0.39 & 5.46 & $\mathrm{Ni}$ & Yes \\
\hline Total: & & & & $\begin{array}{c}100.0 \\
0\end{array}$ & & $\begin{array}{c}100.0 \\
0\end{array}$ & & \\
\hline
\end{tabular}
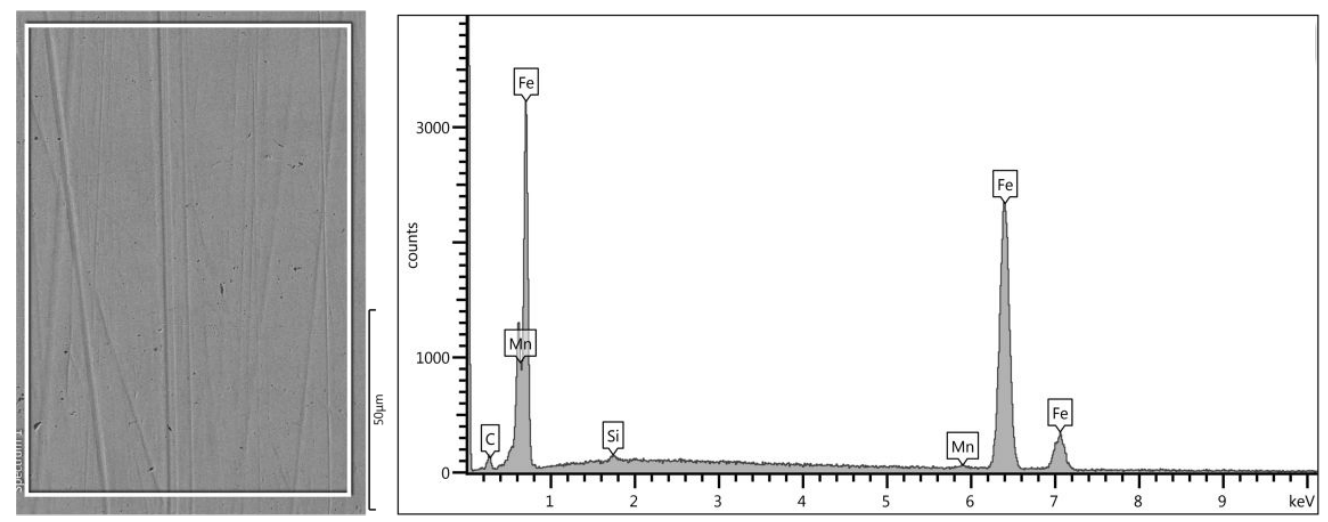

Figure S2. Scanning Electron Microscopy and composition analysis for Steel 304-2B

Table S2. EDS data for Steel 304-2B_A and Steel 304-2B_B 


\begin{tabular}{|c|c|c|c|c|c|c|c|c|}
\hline $\begin{array}{c}\text { Elemen } \\
t\end{array}$ & $\begin{array}{l}\text { Line } \\
\text { Type }\end{array}$ & $\begin{array}{c}\text { Apparent } \\
\text { Concentratio } \\
n\end{array}$ & $\begin{array}{c}\mathrm{k} \\
\text { Ratio }\end{array}$ & Wt $\%$ & $\begin{array}{l}\text { Wt? } \\
\text { Sigm } \\
\text { a }\end{array}$ & $\begin{array}{c}\text { Atomi } \\
\text { c } \div\end{array}$ & $\begin{array}{l}\text { Standar } \\
\text { d Label }\end{array}$ & $\begin{array}{c}\text { Factory } \\
\text { Standar } \\
\text { d }\end{array}$ \\
\hline $\mathrm{C}$ & $\begin{array}{c}\text { K } \\
\text { serie } \\
\text { s }\end{array}$ & 0.14 & $\begin{array}{c}0.0013 \\
6\end{array}$ & 3.46 & 0.35 & 14.26 & C Vit & Yes \\
\hline $\mathrm{Si}$ & $\begin{array}{c}\text { K } \\
\text { serie } \\
\text { s }\end{array}$ & 0.04 & $\begin{array}{c}0.0003 \\
1\end{array}$ & 0.29 & 0.08 & 0.52 & SiO2 & Yes \\
\hline $\mathrm{Mn}$ & $\begin{array}{c}\text { K } \\
\text { serie } \\
\text { s }\end{array}$ & 0.11 & $\begin{array}{c}0.0011 \\
0\end{array}$ & 0.66 & 0.18 & 0.59 & $\mathrm{Mn}$ & Yes \\
\hline $\mathrm{Fe}$ & $\begin{array}{c}K \\
\text { serie } \\
\text { s }\end{array}$ & 16.36 & $\begin{array}{c}0.1635 \\
6\end{array}$ & 95.59 & 0.39 & 84.63 & $\mathrm{Fe}$ & Yes \\
\hline Total: & & & & $\begin{array}{c}100.0 \\
0\end{array}$ & & $\begin{array}{c}100.0 \\
0\end{array}$ & & \\
\hline
\end{tabular}

\begin{tabular}{|c|c|c|c|c|c|c|c|c|}
\hline $\begin{array}{c}\text { Elemen } \\
t\end{array}$ & $\begin{array}{l}\text { Line } \\
\text { Type }\end{array}$ & $\begin{array}{c}\text { Apparent } \\
\text { Concentratio } \\
n\end{array}$ & $\begin{array}{c}\mathrm{k} \\
\text { Ratio }\end{array}$ & Wt $\%$ & $\begin{array}{l}\text { Wt\% } \\
\text { Sigm } \\
\text { a }\end{array}$ & $\begin{array}{c}\text { Atomi } \\
\text { C } \frac{\circ}{0}\end{array}$ & $\begin{array}{l}\text { Standar } \\
\text { d Label }\end{array}$ & $\begin{array}{c}\text { Factory } \\
\text { Standar } \\
\text { d }\end{array}$ \\
\hline $\mathrm{C}$ & $\begin{array}{c}\text { K } \\
\text { serie } \\
\text { s }\end{array}$ & 0.12 & $\begin{array}{c}0.0012 \\
2\end{array}$ & 3.19 & 0.36 & 13.25 & C Vit & Yes \\
\hline $\mathrm{Si}$ & $\begin{array}{c}\text { K } \\
\text { serie } \\
\text { s }\end{array}$ & 0.04 & $\begin{array}{c}0.0003 \\
5\end{array}$ & 0.34 & 0.08 & 0.60 & $\mathrm{SiO} 2$ & Yes \\
\hline $\mathrm{Mn}$ & $\begin{array}{c}\text { K } \\
\text { serie } \\
\text { s }\end{array}$ & 0.12 & $\begin{array}{c}0.0012 \\
2\end{array}$ & 0.74 & 0.18 & 0.68 & $\mathrm{Mn}$ & Yes \\
\hline $\mathrm{Fe}$ & $\begin{array}{c}\mathrm{K} \\
\text { serie } \\
\mathrm{s} \\
\end{array}$ & 16.07 & $\begin{array}{c}0.1607 \\
3\end{array}$ & 95.73 & 0.40 & 85.47 & $\mathrm{Fe}$ & Yes \\
\hline Total: & & & & $\begin{array}{c}100.0 \\
0\end{array}$ & & $\begin{array}{c}100.0 \\
0\end{array}$ & & \\
\hline
\end{tabular}
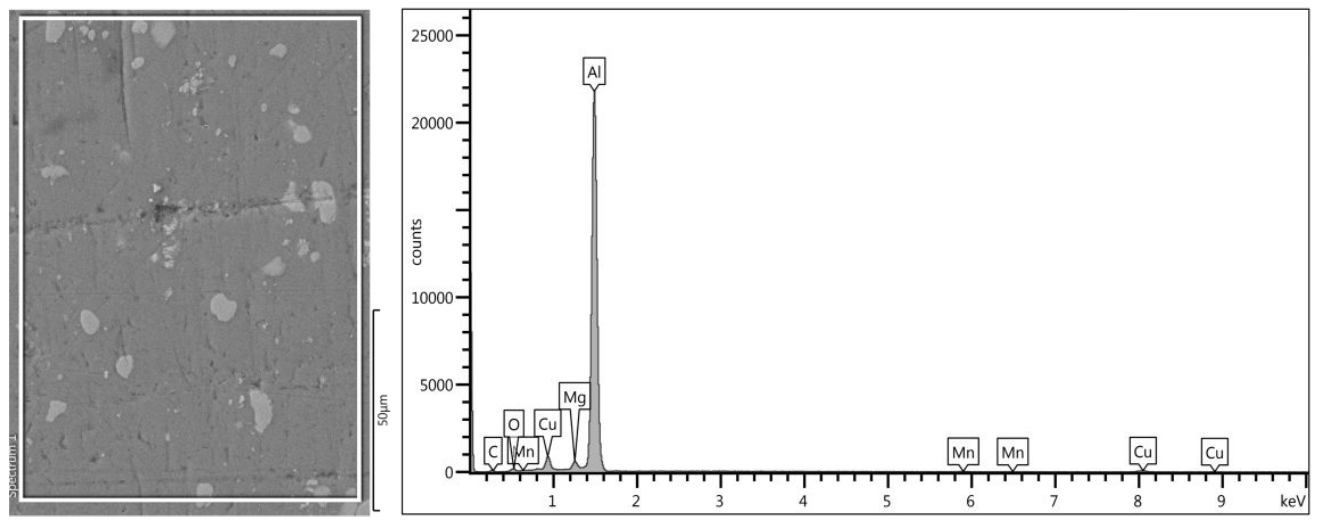

Figure S3. Scanning Electron Microscopy and composition analysis for AA 2024-T3

Table S3. EDS data for AA 2024-T3_A and AA 2024-T3_B

\begin{tabular}{|c|c|c|c|c|c|c|c|c|}
\hline $\begin{array}{c}\text { Elemen } \\
t\end{array}$ & $\begin{array}{c}\text { Line } \\
\text { Type }\end{array}$ & $\begin{array}{c}\text { Apparent } \\
\text { Concentratio } \\
n\end{array}$ & $\begin{array}{c}\mathrm{k} \\
\text { Ratio }\end{array}$ & Wt $\%$ & $\begin{array}{c}\text { Wt } \% \\
\text { Sigm } \\
\mathrm{a}\end{array}$ & $\begin{array}{c}\text { Atomi } \\
\mathrm{c} \%\end{array}$ & $\begin{array}{c}\text { Standar } \\
\mathrm{d} \text { Label }\end{array}$ & $\begin{array}{c}\text { Factory } \\
\text { Standar } \\
\mathrm{d}\end{array}$ \\
\hline
\end{tabular}




\begin{tabular}{|c|c|c|c|c|c|c|c|c|}
\hline $\mathrm{C}$ & $\begin{array}{c}\text { K } \\
\text { serie } \\
\text { s }\end{array}$ & 0.05 & $\begin{array}{c}0.0004 \\
9\end{array}$ & 3.23 & 0.59 & 7.06 & C Vit & Yes \\
\hline 0 & $\begin{array}{c}\text { K } \\
\text { serie } \\
\text { s }\end{array}$ & 0.34 & $\begin{array}{c}0.0011 \\
5\end{array}$ & 2.17 & 0.17 & 3.57 & $\mathrm{SiO} 2$ & Yes \\
\hline $\mathrm{Mg}$ & $\begin{array}{c}\text { K } \\
\text { serie } \\
\text { s }\end{array}$ & 0.31 & $\begin{array}{c}0.0020 \\
6\end{array}$ & 1.33 & 0.07 & 1.44 & $\mathrm{MgO}$ & Yes \\
\hline Al & $\begin{array}{c}\text { K } \\
\text { serie } \\
\text { s }\end{array}$ & 19.24 & $\begin{array}{c}0.1381 \\
7\end{array}$ & 88.22 & 0.68 & 85.80 & A1203 & Yes \\
\hline $\mathrm{Mn}$ & $\begin{array}{c}\text { K } \\
\text { serie } \\
\text { s }\end{array}$ & 0.12 & $\begin{array}{c}0.0011 \\
6\end{array}$ & 0.79 & 0.15 & 0.38 & $\mathrm{Mn}$ & Yes \\
\hline $\mathrm{Cu}$ & $\begin{array}{c}\text { K } \\
\text { serie } \\
\text { s }\end{array}$ & 0.61 & $\begin{array}{c}0.0061 \\
3\end{array}$ & 4.25 & 0.38 & 1.75 & $\mathrm{Cu}$ & Yes \\
\hline Total: & & & & $\begin{array}{c}100.0 \\
0\end{array}$ & & $\begin{array}{c}100.0 \\
0\end{array}$ & & \\
\hline
\end{tabular}

\begin{tabular}{|c|c|c|c|c|c|c|c|c|}
\hline $\begin{array}{c}\text { Elemen } \\
t\end{array}$ & $\begin{array}{l}\text { Line } \\
\text { Type }\end{array}$ & $\begin{array}{c}\text { Apparent } \\
\text { Concentratio } \\
n\end{array}$ & $\begin{array}{c}\mathrm{k} \\
\text { Ratio }\end{array}$ & wt $\%$ & $\begin{array}{l}\text { wt } \frac{0}{0} \\
\text { Sigm } \\
\text { a }\end{array}$ & $\begin{array}{c}\text { Atomi } \\
\text { C } \frac{\circ}{0}\end{array}$ & $\begin{array}{l}\text { Standar } \\
\text { d Label }\end{array}$ & $\begin{array}{c}\text { Factory } \\
\text { Standar } \\
\text { d }\end{array}$ \\
\hline $\mathrm{C}$ & $\begin{array}{c}\text { K } \\
\text { serie } \\
\text { s }\end{array}$ & 0.09 & $\begin{array}{c}0.0009 \\
5\end{array}$ & 5.77 & 0.63 & 12.40 & C Vit & Yes \\
\hline 0 & $\begin{array}{c}\text { K } \\
\text { serie } \\
\text { s }\end{array}$ & 0.27 & $\begin{array}{c}0.0009 \\
0\end{array}$ & 1.64 & 0.15 & 2.65 & $\mathrm{SiO} 2$ & Yes \\
\hline $\mathrm{Mg}$ & $\begin{array}{c}\mathrm{K} \\
\text { serie } \\
\mathrm{s} \\
\end{array}$ & 0.37 & $\begin{array}{c}0.0024 \\
5\end{array}$ & 1.58 & 0.07 & 1.68 & $\mathrm{MgO}$ & Yes \\
\hline $\mathrm{Al}$ & $\begin{array}{c}\text { K } \\
\text { serie } \\
\text { s } \\
\end{array}$ & 18.65 & $\begin{array}{c}0.1339 \\
2\end{array}$ & 84.04 & 0.69 & 80.40 & Al203 & Yes \\
\hline $\mathrm{Mn}$ & $\begin{array}{c}\mathrm{K} \\
\text { serie } \\
\mathrm{s} \\
\end{array}$ & 0.10 & $\begin{array}{c}0.0009 \\
6\end{array}$ & 0.61 & 0.14 & 0.29 & $\mathrm{Mn}$ & Yes \\
\hline $\mathrm{Cu}$ & $\begin{array}{c} \\
\text { serie } \\
\text { s } \\
\end{array}$ & 0.97 & $\begin{array}{c}0.0096 \\
8\end{array}$ & 6.36 & 0.39 & 2.58 & $\mathrm{Cu}$ & Yes \\
\hline Total: & & & & $\begin{array}{c}100.0 \\
0\end{array}$ & & $\begin{array}{c}100.0 \\
0\end{array}$ & & \\
\hline
\end{tabular}

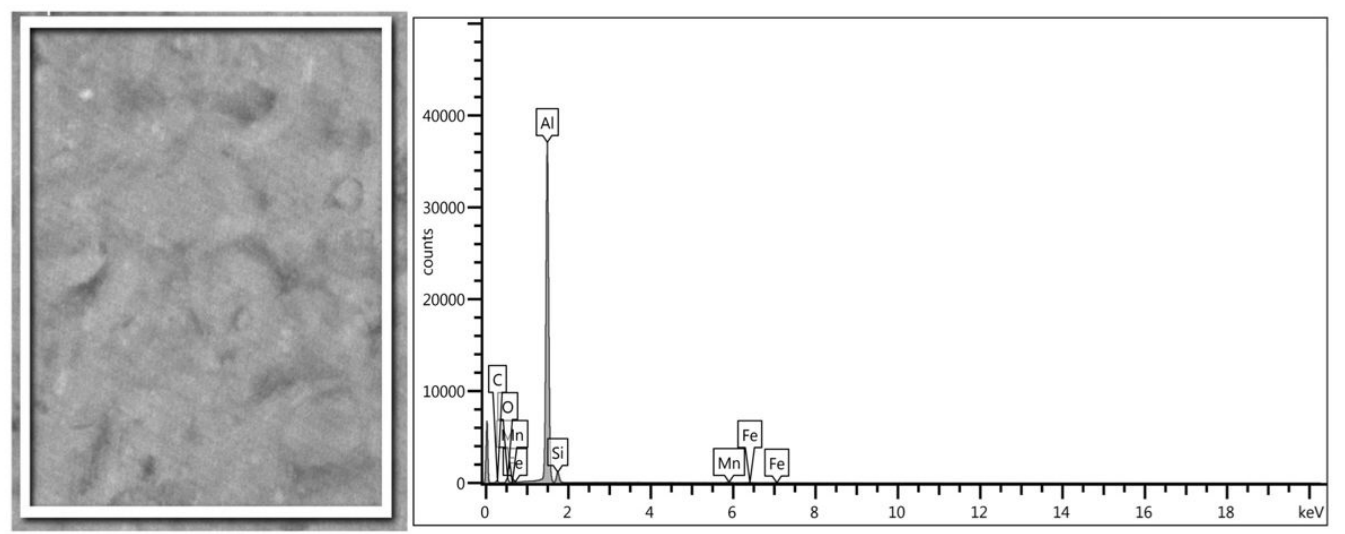


Figure S4. Scanning Electron Microscopy and composition analysis for AA 3003-H14

Table S4. EDS data for AA 3003-H14

\begin{tabular}{|c|c|c|c|c|c|c|c|}
\hline Element & $\begin{array}{l}\text { Line } \\
\text { Type }\end{array}$ & $\begin{array}{c}\text { Apparent } \\
\text { Concentration }\end{array}$ & k Ratio & wt\% & $\begin{array}{c}\text { wt\% } \\
\text { Sigma }\end{array}$ & $\begin{array}{c}\text { Standard } \\
\text { Label }\end{array}$ & $\begin{array}{l}\text { Factory } \\
\text { Standard }\end{array}$ \\
\hline C & $\begin{array}{c}\mathrm{K} \\
\text { series }\end{array}$ & 1.03 & 0.01028 & 4.19 & 0.23 & C Vit & Yes \\
\hline 0 & & & & 51.13 & & & \\
\hline $\mathrm{Al}$ & $\begin{array}{c}\text { K } \\
\text { series }\end{array}$ & 80.92 & 0.58121 & 41.98 & 0.22 & Al203 & Yes \\
\hline $\mathrm{Si}$ & $\begin{array}{c}\mathrm{K} \\
\text { series }\end{array}$ & 2.49 & 0.01975 & 2.17 & 0.04 & SiO2 & Yes \\
\hline $\mathrm{Mn}$ & $\begin{array}{c}\mathrm{K} \\
\text { series } \\
\end{array}$ & 0.63 & 0.00634 & 0.37 & 0.03 & $\mathrm{Mn}$ & Yes \\
\hline $\mathrm{Fe}$ & $\begin{array}{c}\mathrm{K} \\
\text { series }\end{array}$ & 0.29 & 0.00288 & 0.16 & 0.03 & $\mathrm{Fe}$ & Yes \\
\hline Total: & & & & 100.00 & & & \\
\hline
\end{tabular}

Evaluation of the coupons were carried out employing Brines 1 to 4. General dimensions for the coupons are: a. stainless steel 304-2B (50mm x 10mm x 0.45mm), b. AA 3003-H14 probes $(50 \mathrm{~mm} \times 10 \mathrm{~mm} \times 0.43 \mathrm{~mm})$ c. carbon steel $1045(50 \mathrm{~mm} \times 10 \mathrm{~mm} \times 2.2 \mathrm{~mm})$ and $\mathrm{d}$. aluminum alloy 2024-T3 (57 $\mathrm{mm}$ x 9mm x 2.0mm). Coupons were hanging from a brass wire, which was welded and coated with and insulating coating from the probe's contact to the top to avoid conduction current. Therefore, exposed area to scaling environment was reduced. Table 6_SI exhibit the most relevant information. 


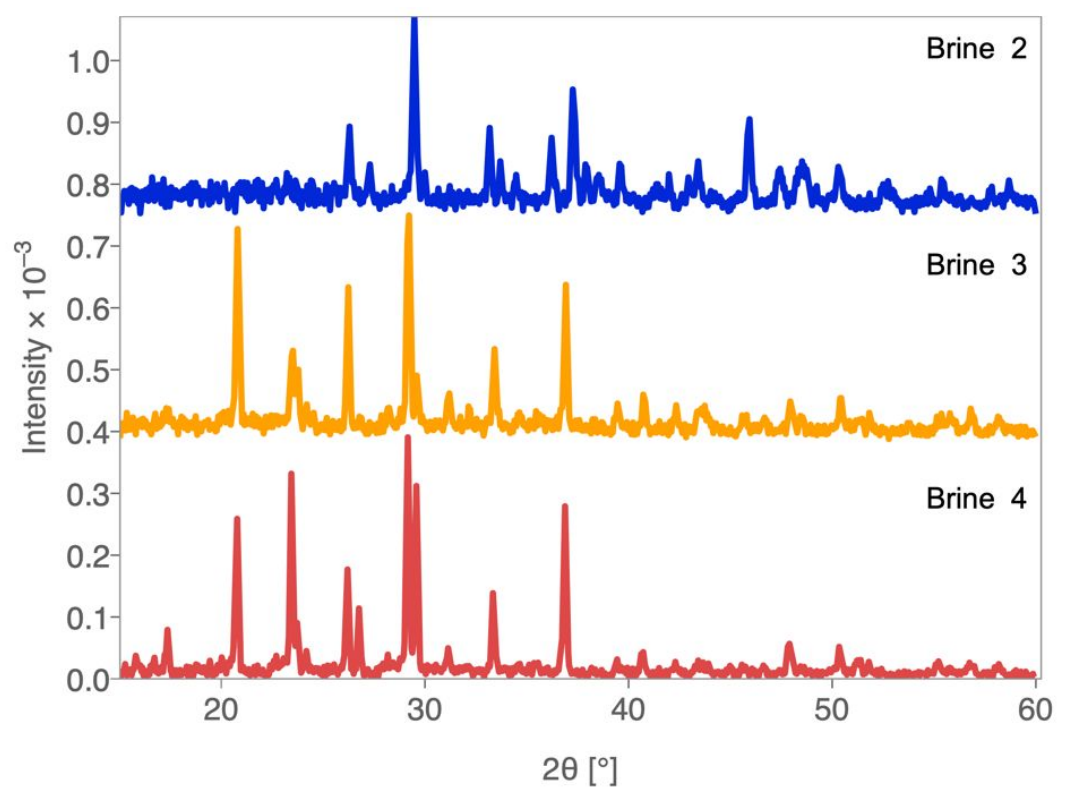

Figure S5. PXRD of scales growth from Brine 2, 3, and $\mathbf{4}$ for 304-2B and 3003-H14 coupons.

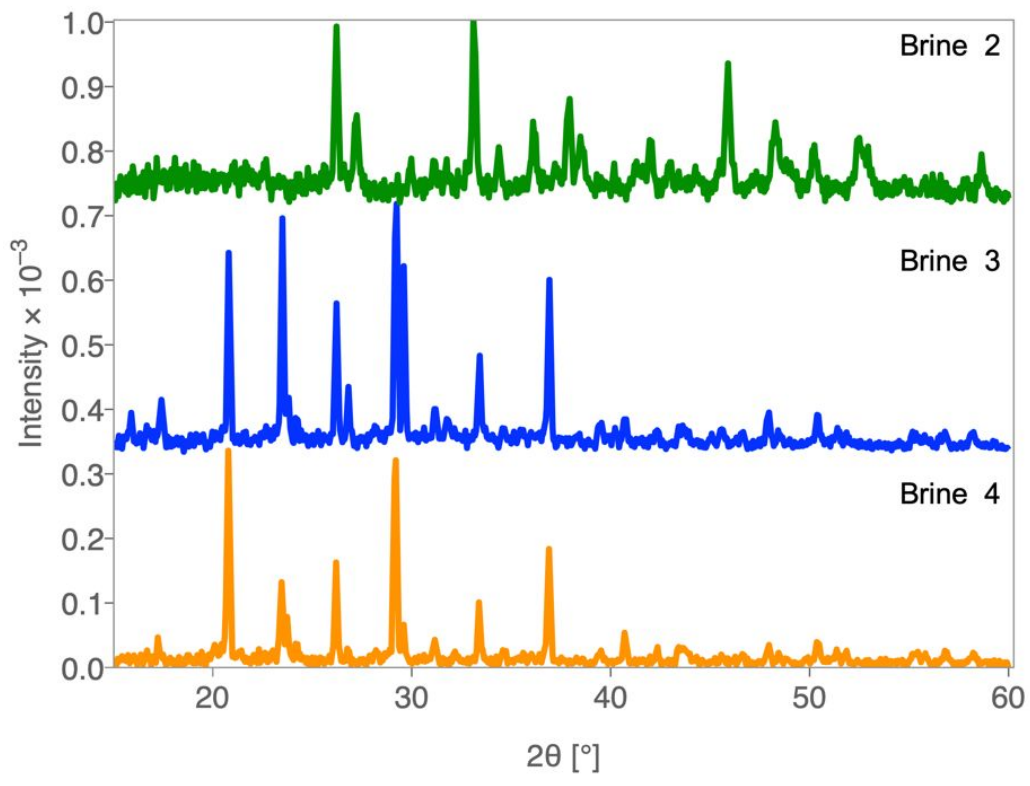

Figure S6. PXRD of scales growth from Brine 2, 3, and 4 for 1045 and 2024-T3 coupons. 


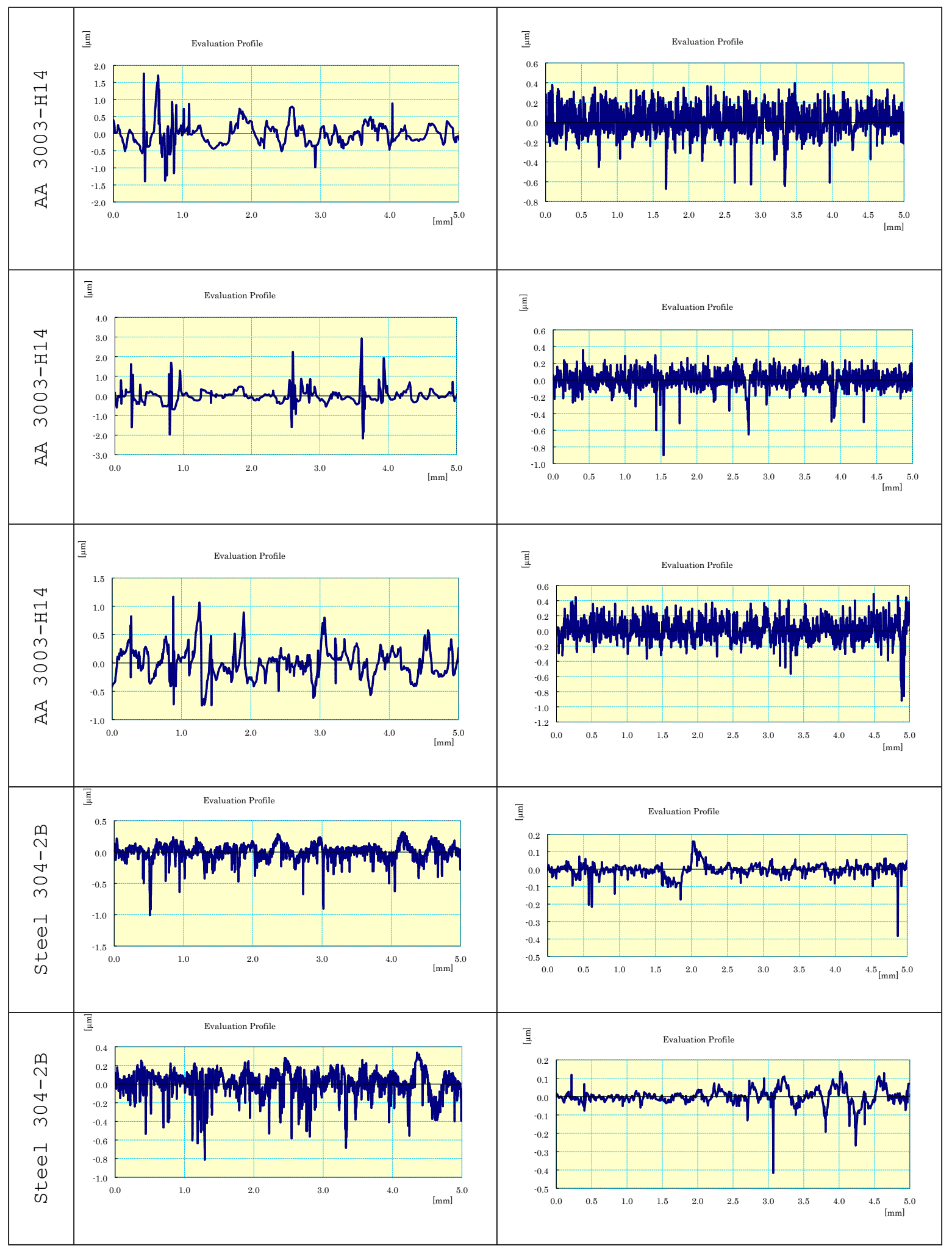




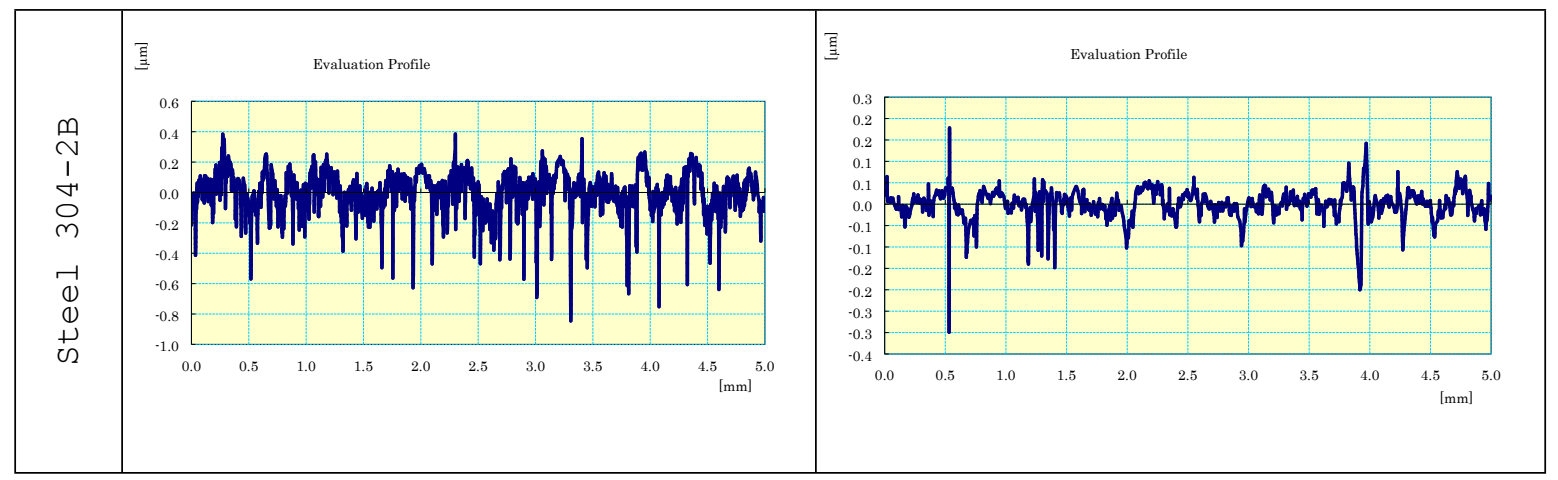

Figure S7. Mechanical profilometry diagrams for AA 3003-H14 and Steel 304-2B before and after grounding.

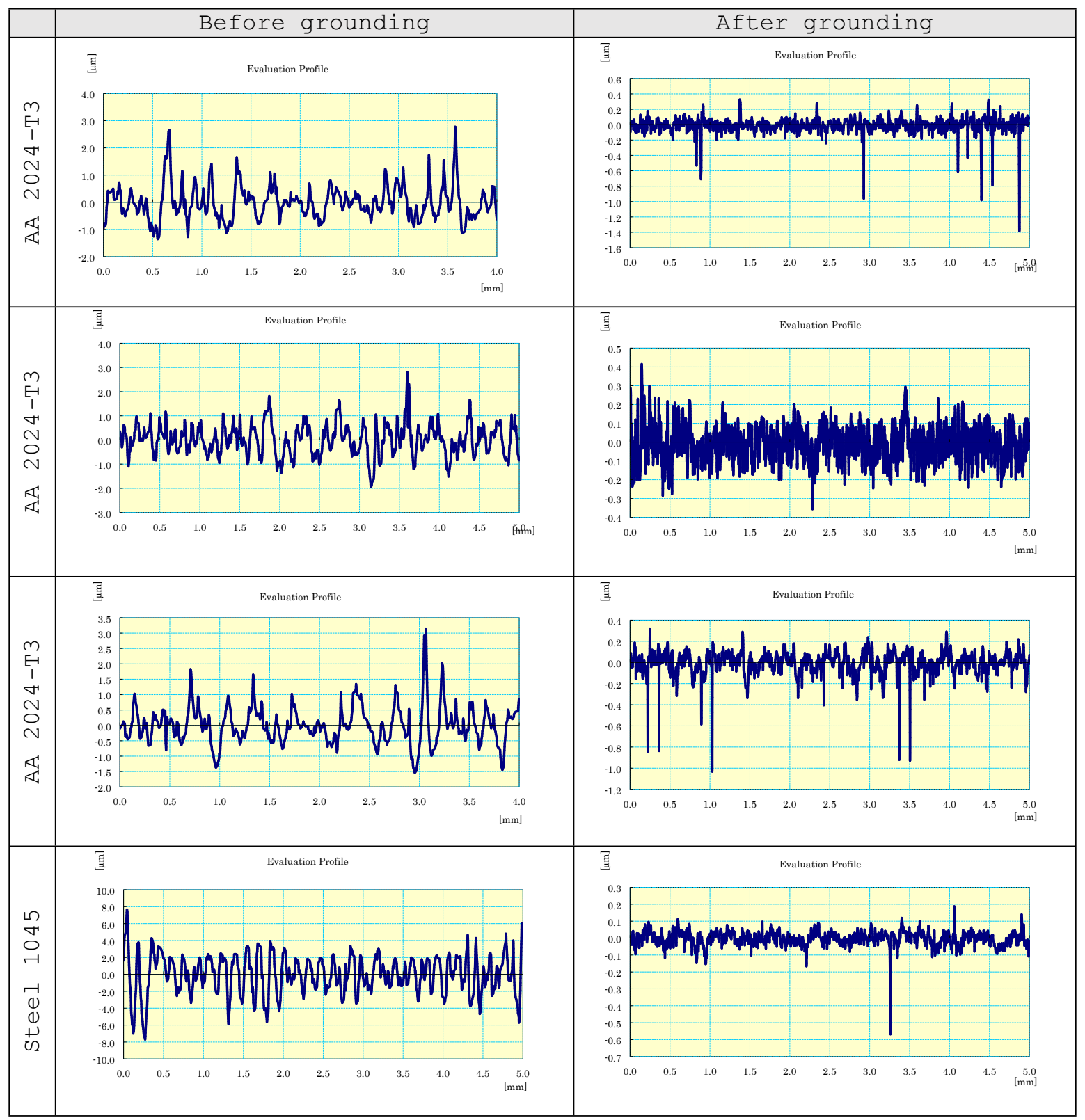




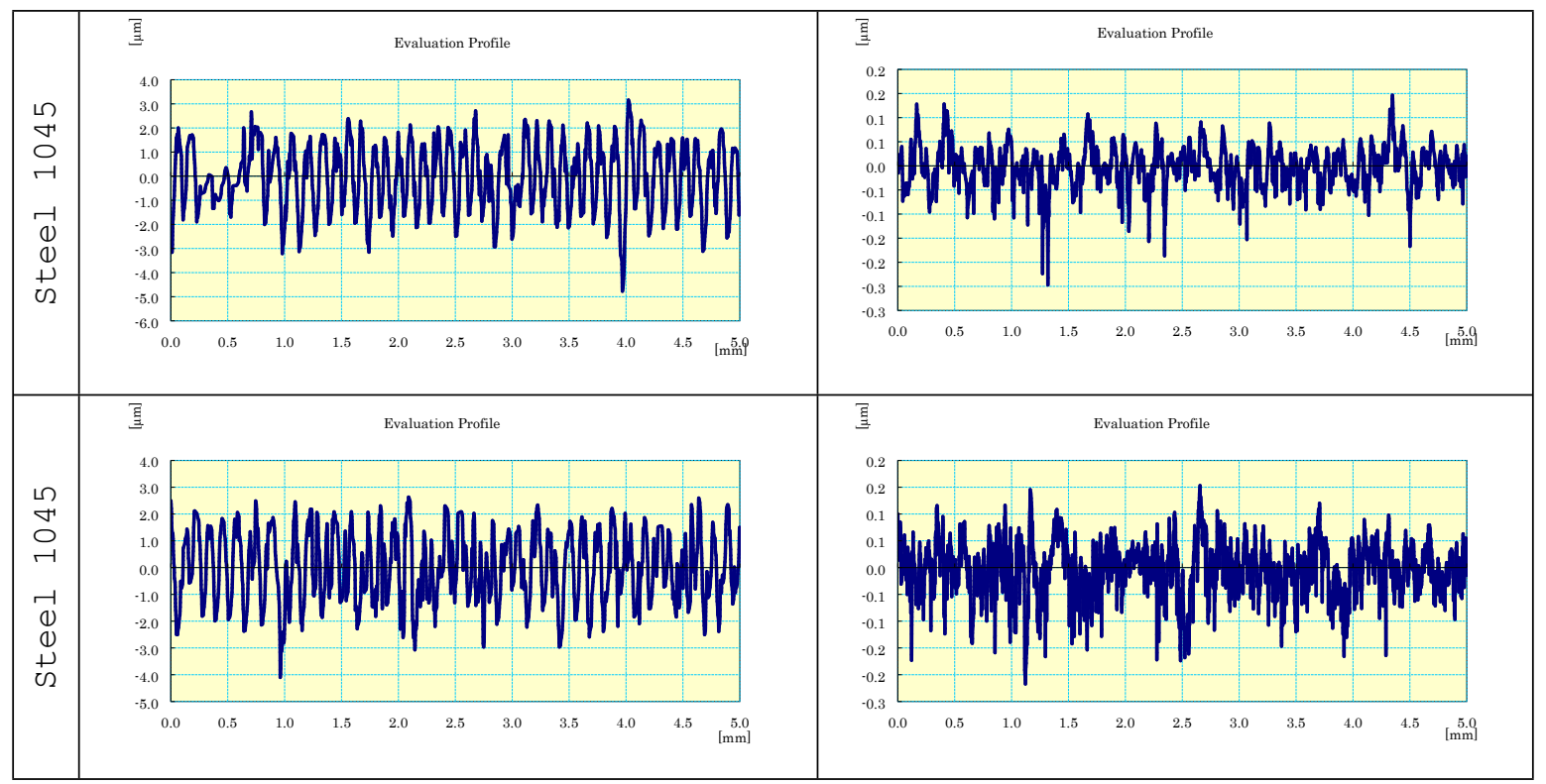

Figure S8. Mechanical profilometry diagrams for AA 2024-T3 and Steel 1045 before and after grounding.

Table S5. Mechanical profilometry results before immersion in scaling environments (Brines 2-4).

\begin{tabular}{|c|c|c|c|c|c|}
\hline $\begin{array}{c}\text { Steel } \\
304-\mathrm{B} 2\end{array}$ & $\begin{array}{c}\text { Before } \\
\text { grounding } \\
(\mathrm{Ra} ; \mu \mathrm{m})\end{array}$ & $\begin{array}{c}\text { After } \\
\text { grounding } \\
(\mathrm{Ra} ; \mu \mathrm{m})\end{array}$ & $\begin{array}{c}\text { AA } 3003- \\
\mathrm{H} 14\end{array}$ & $\begin{array}{c}\text { Before } \\
\text { grounding } \\
(\mathrm{Ra} ; \mu \mathrm{m})\end{array}$ & $\begin{array}{c}\text { After } \\
\text { grounding } \\
(\mathrm{Ra} ; \mu \mathrm{m})\end{array}$ \\
\hline $\mathrm{A}$ & 0.105 & 0.022 & $\mathrm{~A}$ & 0.246 & 0.098 \\
\hline $\mathrm{B}$ & 0.099 & 0.028 & $\mathrm{~B}$ & 0.251 & 0.094 \\
\hline $\mathrm{C}$ & 0.100 & 0.023 & $\mathrm{C}$ & 0.260 & 0.098 \\
\hline $\begin{array}{c}\text { Steel } \\
1045\end{array}$ & & & $\mathrm{AA} 2024-$ & & \\
\hline $\mathrm{A}$ & 1.855 & 0.030 & $\mathrm{~A}$ & 0.462 & 0.066 \\
\hline $\mathrm{B}$ & 1.175 & 0.032 & $\mathrm{~B}$ & 0.446 & 0.077 \\
\hline $\mathrm{C}$ & 1.119 & 0.038 & C & 0.212 & 0.076 \\
\hline
\end{tabular}

Table S6. Coupons' exposed area and scale mass for environment brines 2, 3, and 4. 
2. CALCIUM CARBONATE

\begin{tabular}{|c|c|c|c|c|c|c|c|}
\hline & Sample & $\begin{array}{c}\text { Lengt } \\
\mathbf{h} \\
(\mathbf{c m})\end{array}$ & $\begin{array}{c}\text { Width } \\
\mathbf{( c m )}\end{array}$ & $\begin{array}{c}\text { Thickne } \\
\mathbf{s s}(\mathbf{c m})\end{array}$ & $\begin{array}{c}\text { Exposed } \\
\text { area }\left(\mathbf{c m}^{2}\right)\end{array}$ & $\begin{array}{c}\text { Accumulat } \\
\text { ed Mass } \\
(\mathbf{g})\end{array}$ & $\begin{array}{c}\text { Scale } \\
\mathbf{m a s s} \\
\left(\mathbf{k g} / \mathbf{m}^{2}\right)\end{array}$ \\
\hline $\mathbf{1}$ & $\mathbf{3 0 4 - 2 B}$ & 3.1 & 1 & 0.036 & 6.4592 & 0.013 & $\begin{array}{c}0.020126 \\
331\end{array}$ \\
\hline $\mathbf{2}$ & $\mathbf{1 0 4 5}$ & 4.3 & 1 & 0.205 & 10.5680 & 0.054 & $\begin{array}{c}0.051097 \\
653\end{array}$ \\
\hline $\mathbf{3}$ & $\mathbf{2 0 2 4 - T 3}$ & 5 & 0.9 & 0.188 & 11.0492 & 0.061 & $\begin{array}{c}0.055207 \\
617\end{array}$ \\
\hline $\mathbf{4}$ & $\begin{array}{c}\mathbf{3 0 0 3 -} \\
\mathbf{H 1 4}\end{array}$ & 2.9 & 1 & 0.041 & 6.0788 & 0.162 & $\begin{array}{c}0.266499 \\
967\end{array}$ \\
\hline
\end{tabular}

\begin{tabular}{|c|c|c|c|c|c|c|c|}
\hline \multicolumn{7}{|c|}{ 3. CALCIUM SULFATE } \\
\hline & Sample & $\begin{array}{c}\text { Length } \\
(\mathbf{c m})\end{array}$ & $\begin{array}{c}\text { Width } \\
(\mathbf{c m})\end{array}$ & $\begin{array}{c}\text { Thickne } \\
\text { ss (cm) }\end{array}$ & $\begin{array}{c}\text { Exposed } \\
\text { area } \\
\left(\mathbf{c m}^{2}\right)\end{array}$ & $\begin{array}{c}\text { Accumulate } \\
\text { d Mass (g) }\end{array}$ & $\begin{array}{c}\text { Scale } \\
\text { mass } \\
\left(\mathbf{k g} / \mathrm{m}^{2}\right)\end{array}$ \\
\hline $\mathbf{1}$ & $\mathbf{3 0 4 - 2 B}$ & 3.1 & 1 & 0.051 & 6.5672 & 0.006 & $\begin{array}{c}0.001363 \\
14\end{array}$ \\
\hline $\mathbf{2}$ & $\mathbf{1 0 4 5}$ & 4.3 & 1 & 0.197 & 10.4912 & 0.060 & $\begin{array}{c}0.0571907 \\
88\end{array}$ \\
\hline $\mathbf{3}$ & $\mathbf{2 0 2 4 - T 3}$ & 4.9 & 0.9 & 0.179 & 10.7353 & 0.058 & $\begin{array}{c}0.0540273 \\
68\end{array}$ \\
\hline $\mathbf{4}$ & $\begin{array}{c}\mathbf{3 0 0 3 -} \\
\text { H14 }\end{array}$ & 3.1 & 1 & 0.045 & 6.5240 & 0.160 & $\begin{array}{c}0.2452483 \\
14\end{array}$ \\
\hline
\end{tabular}

\begin{tabular}{|c|c|c|c|c|c|c|c|}
\hline \multicolumn{8}{|c|}{ 4. SULFATES + CARBONATES } \\
\hline & Sample & $\begin{array}{l}\text { Length } \\
\text { (cm) }\end{array}$ & $\begin{array}{l}\text { Width } \\
(\mathrm{cm})\end{array}$ & $\begin{array}{l}\text { Thickne } \\
\text { ss (cm) }\end{array}$ & $\begin{array}{c}\text { Exposed } \\
\text { area } \\
\left(\mathrm{cm}^{2}\right)\end{array}$ & $\begin{array}{l}\text { Accumulate } \\
\text { d Mass }(g)\end{array}$ & $\begin{array}{c}\text { Scale } \\
\text { mass } \\
\left(\mathrm{kg} / \mathrm{m}^{2}\right)\end{array}$ \\
\hline 1 & $304-2 B$ & 2.9 & 1 & 0.048 & 6.1264 & 0.003 & $\begin{array}{c}0.0048968 \\
4\end{array}$ \\
\hline 2 & 1045 & 4.1 & 1 & 0.205 & 10.0860 & 0.063 & $\begin{array}{c}0.0624628 \\
2\end{array}$ \\
\hline 3 & $2024-\mathrm{T} 3$ & 4.9 & 0.9 & 0.191 & 10.8637 & 0.092 & $\begin{array}{c}0.0838597 \\
35\end{array}$ \\
\hline 4 & $\begin{array}{c}3003- \\
\text { H14 }\end{array}$ & 3.0 & 1 & 0.042 & 6.2940 & 0.144 & $\begin{array}{c}0.2287893 \\
23 \\
\end{array}$ \\
\hline
\end{tabular}



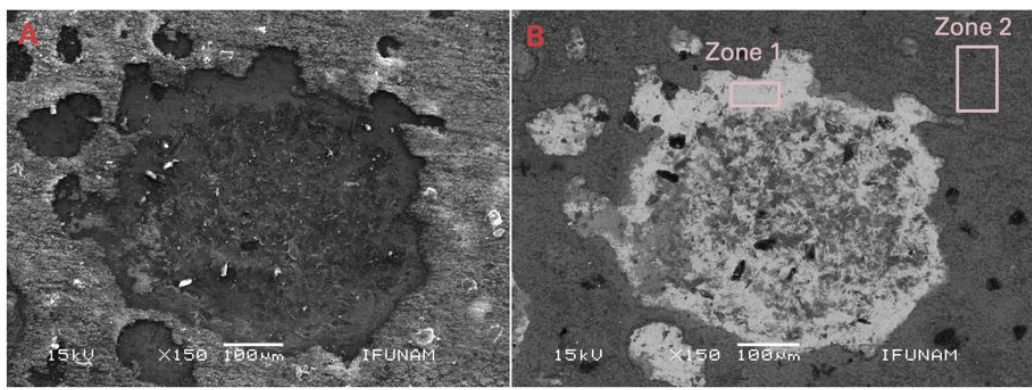

\begin{tabular}{|ccc|}
\hline Element & Zone 1 & Zone 2 \\
\hline $\mathbf{C}$ & 15.15 & 17.39 \\
$\mathbf{O}$ & 1.38 & 30.73 \\
$\mathbf{S i}$ & 0.72 & 0.83 \\
$\mathbf{F e}$ & 79.51 & 4.38 \\
$\mathbf{C l}$ & 0.22 & 0.94 \\
$\mathbf{N a}$ & 2.30 & 3.38 \\
$\mathbf{C a}$ & 0.73 & 41.66 \\
\hline
\end{tabular}
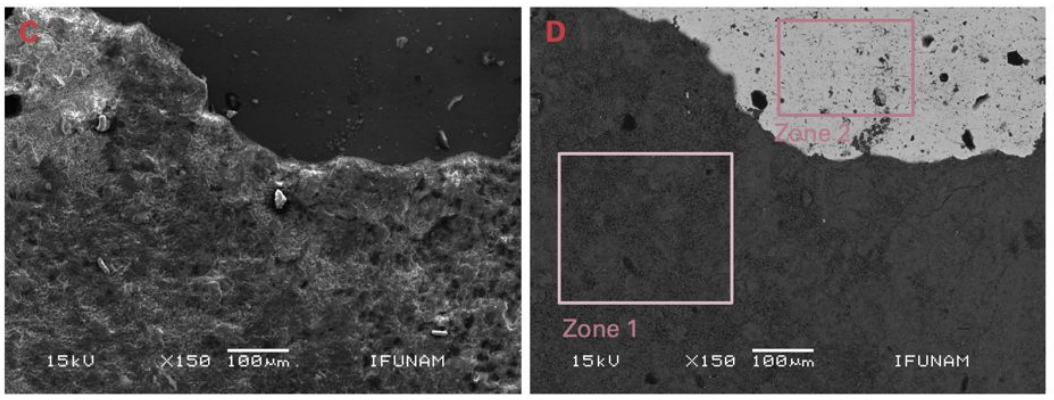

\begin{tabular}{ccc} 
Element & Zone 1 & Zone 2 \\
\hline $\mathbf{C}$ & 9.20 & 5.81 \\
$\mathbf{S i}$ & 0.54 & 0.92 \\
$\mathbf{M n}$ & - & 0.85 \\
$\mathbf{F e}$ & - & 67.26 \\
$\mathbf{O}$ & 28.82 & - \\
$\mathbf{C r}$ & - & 17.62 \\
$\mathbf{N a}$ & 2.69 & - \\
\hline
\end{tabular}
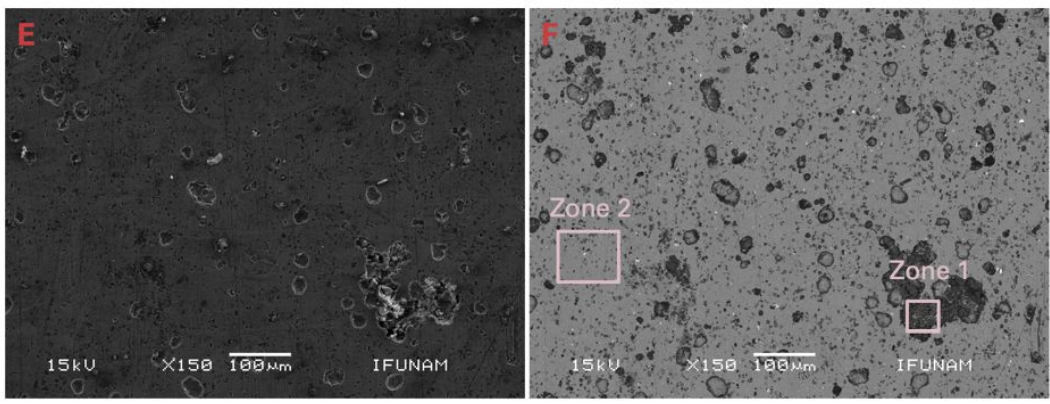

\section{Element 10}
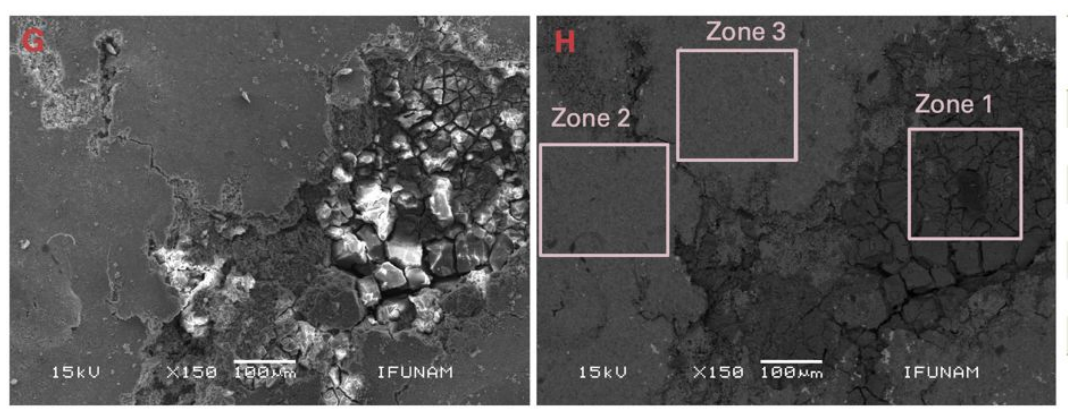

Element Zone 1 Zone 2 Zone 3

Figure S9. Backscattered electron micrographs for A: 1045, C: 304-2B, E: 2024-T3, G: 3003-H14 and secondary electron micrographs for B: 1045, D: 304-2B, F: 2024-T3, H:

3003-H14 


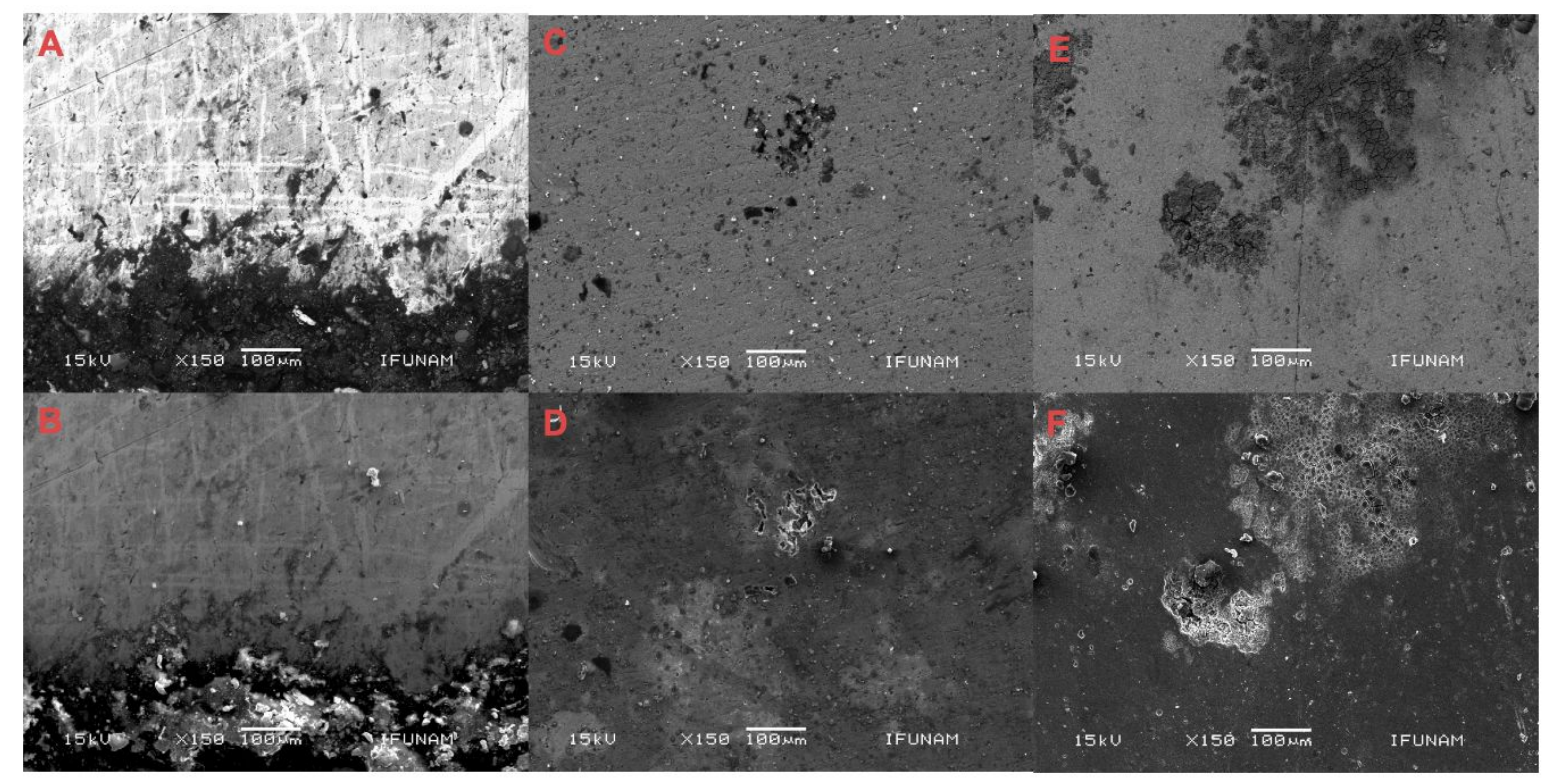

Figure S10. Backscattered electron micrographs for base line experiments, non-scaling; A: 304-2B, C: 2024-T3, E: 3003-H14 and secondary electron micrographs for B: 304-2B, D: 2024-T3, F: 3003-H14. 


\section{Brine 2}
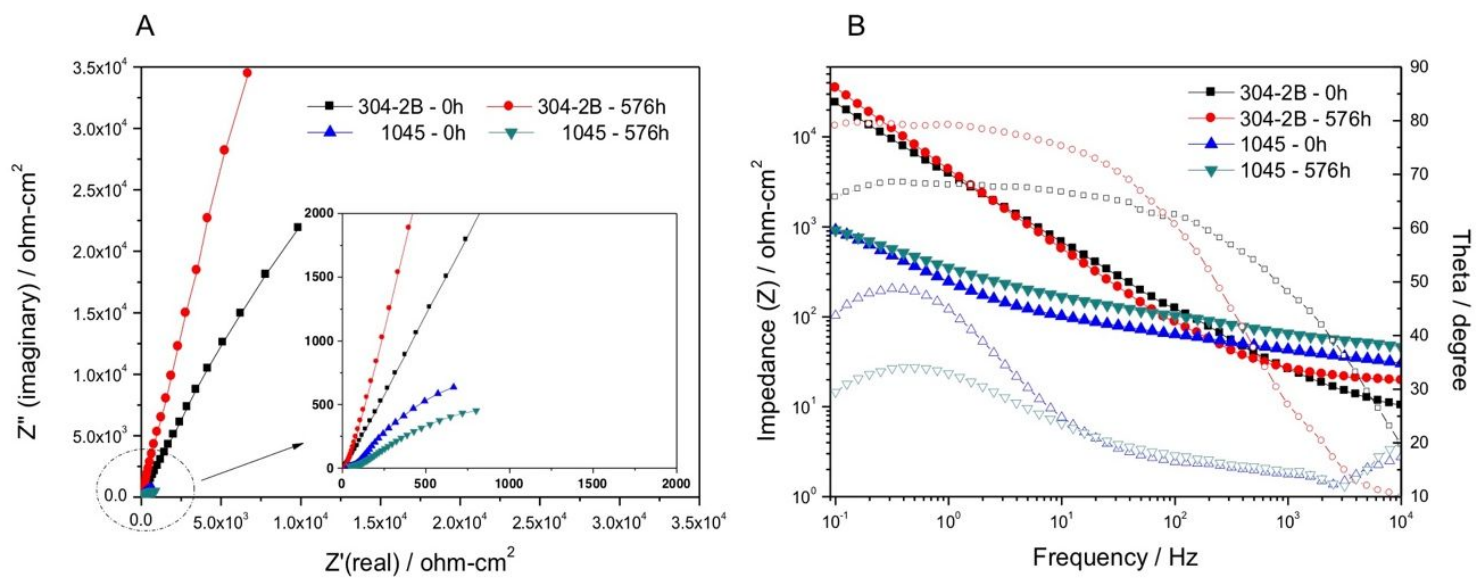

\section{Brine 3}
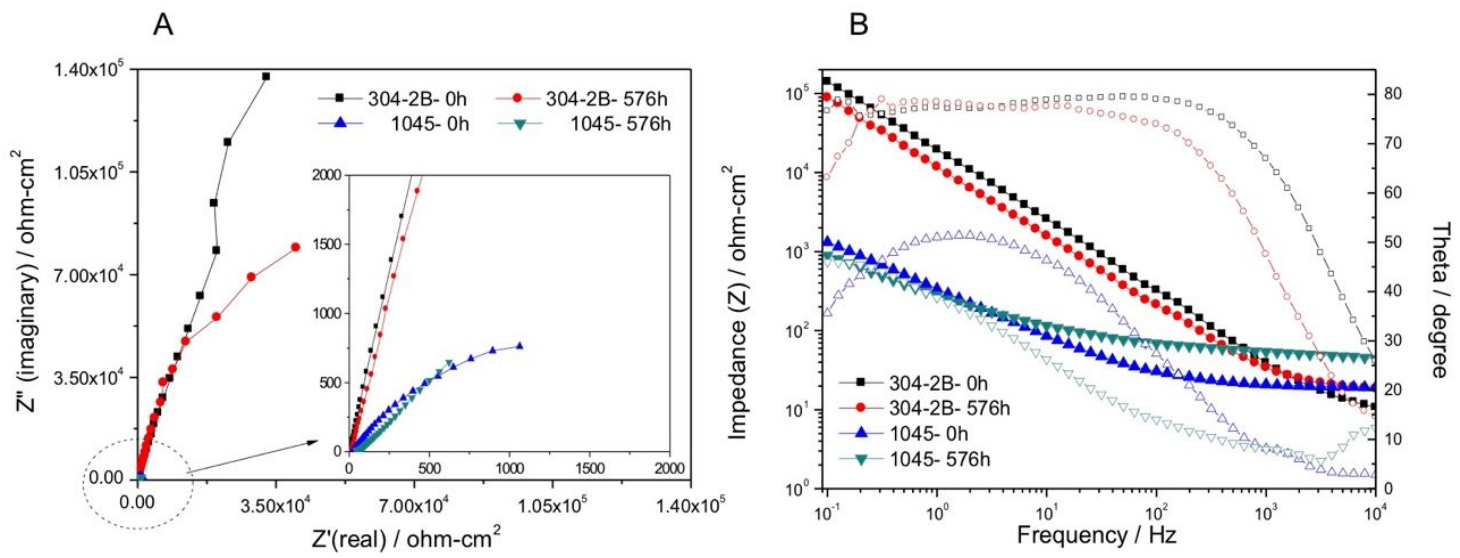

\section{Brine 4}
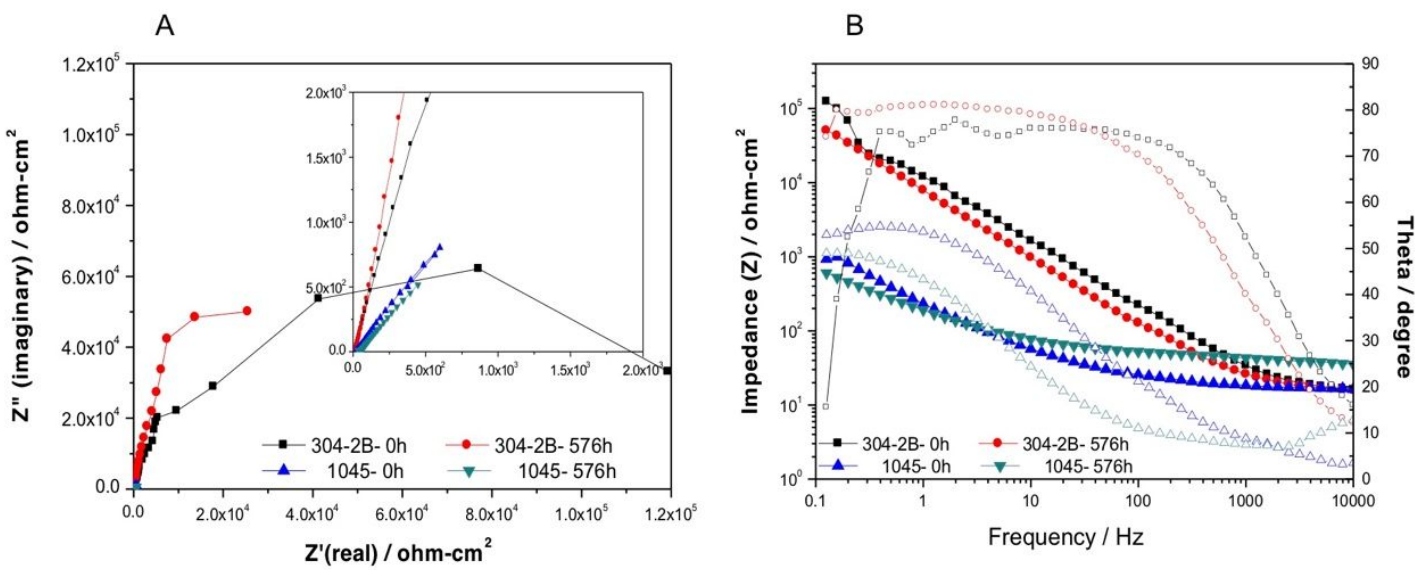

Figure S11. EIS data for 304-2B and 1045 at $0 \mathrm{~h}$ and $576 \mathrm{~h}$ of testing: Nyquist (A) and Bode (B) 


\section{Brine 2}
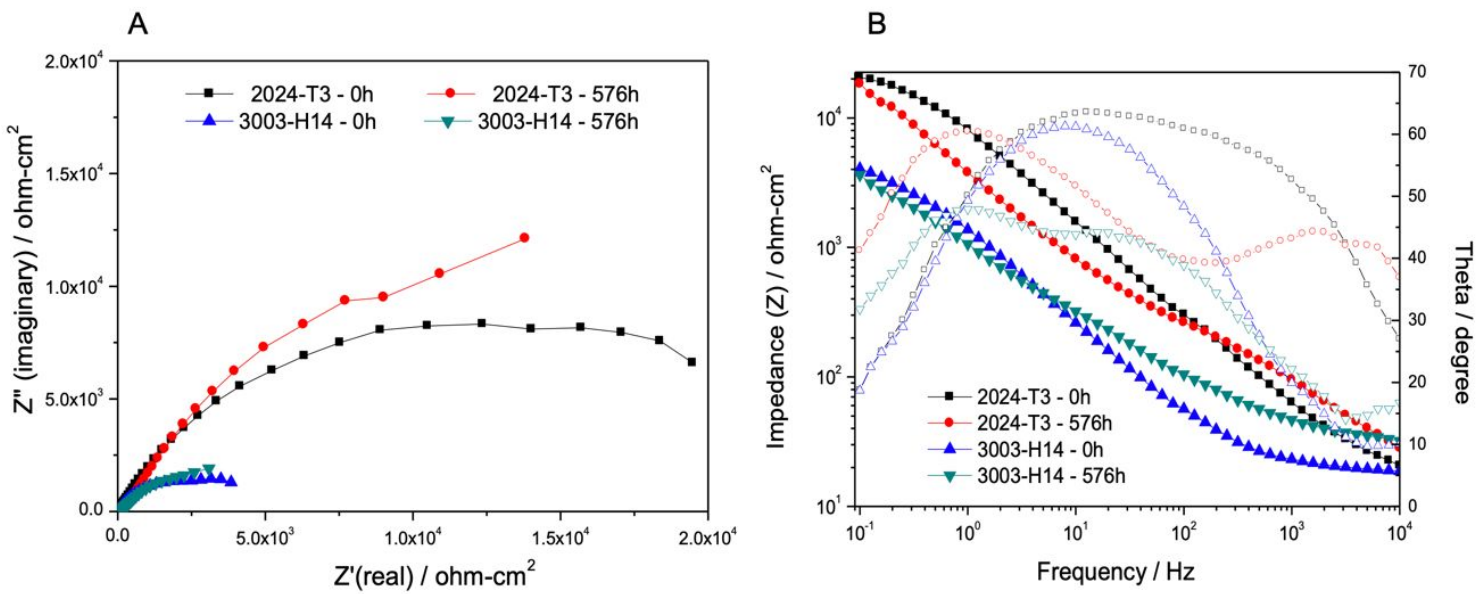

Brine 3
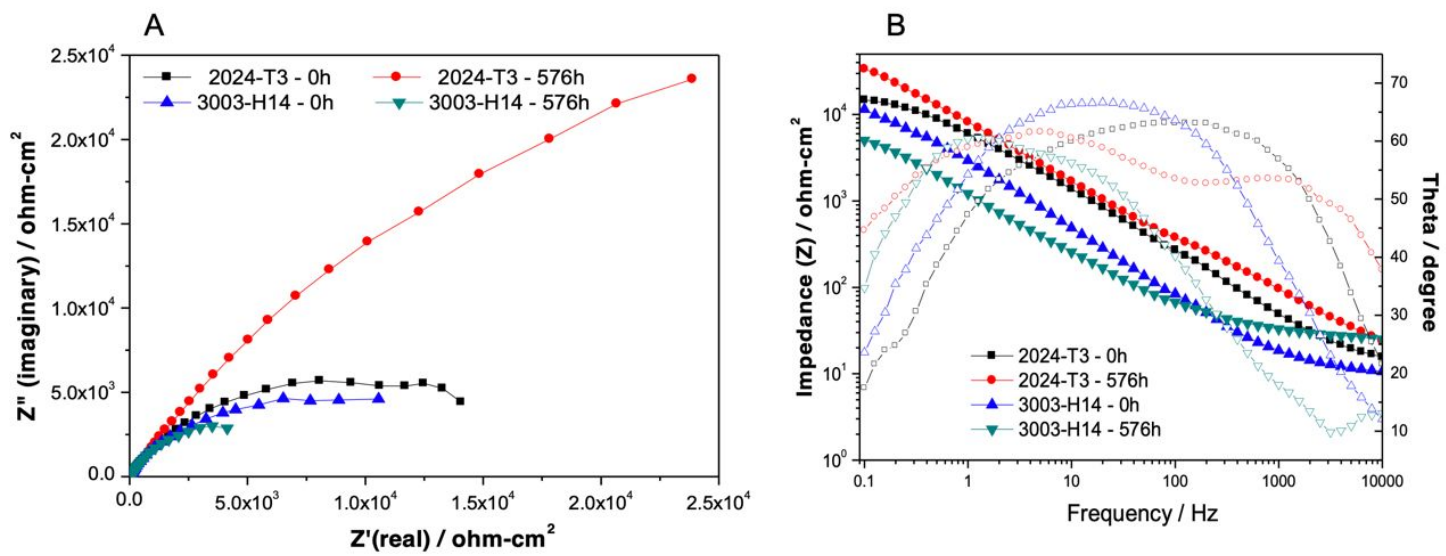

\section{Brine 4}
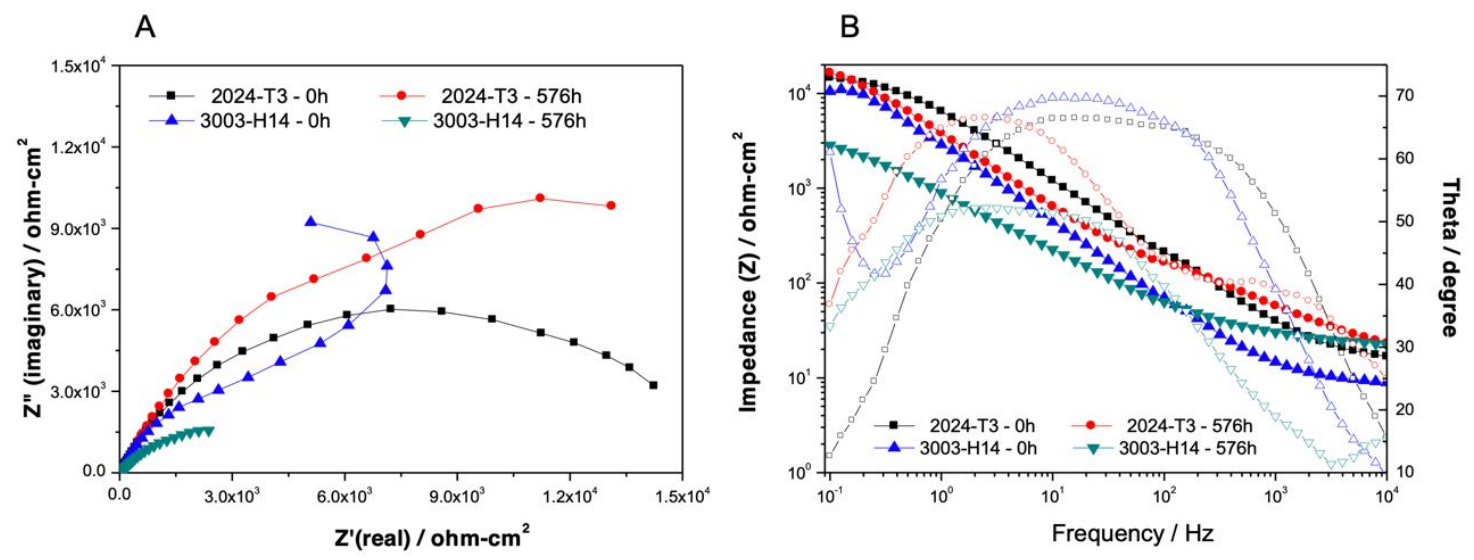

Figure S12. EIS data for 3003-H14 and 2024-T3 at $0 \mathrm{~h}$ and $576 \mathrm{~h}$ of testing: Nyquist (A) and Bode (B) 

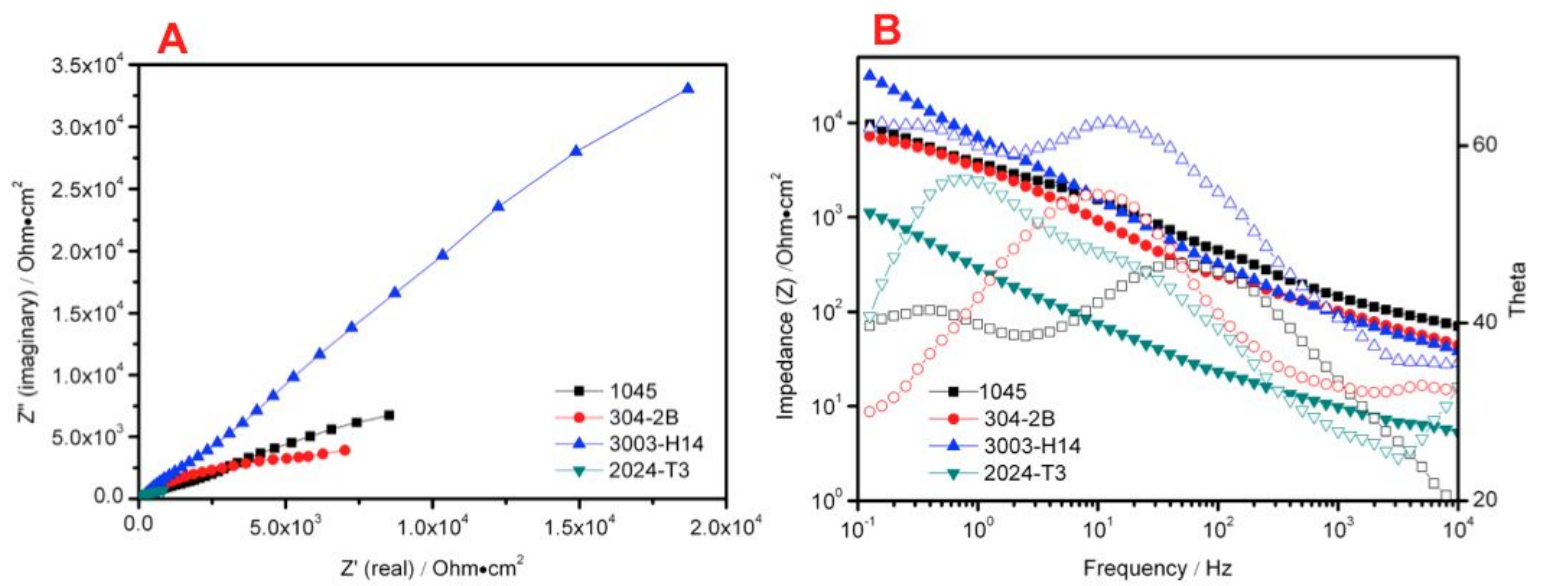

Figure S13. EIS data for base line experiments (non-scaling environment, Brine 1): Nyquist (A) and Bode (B)
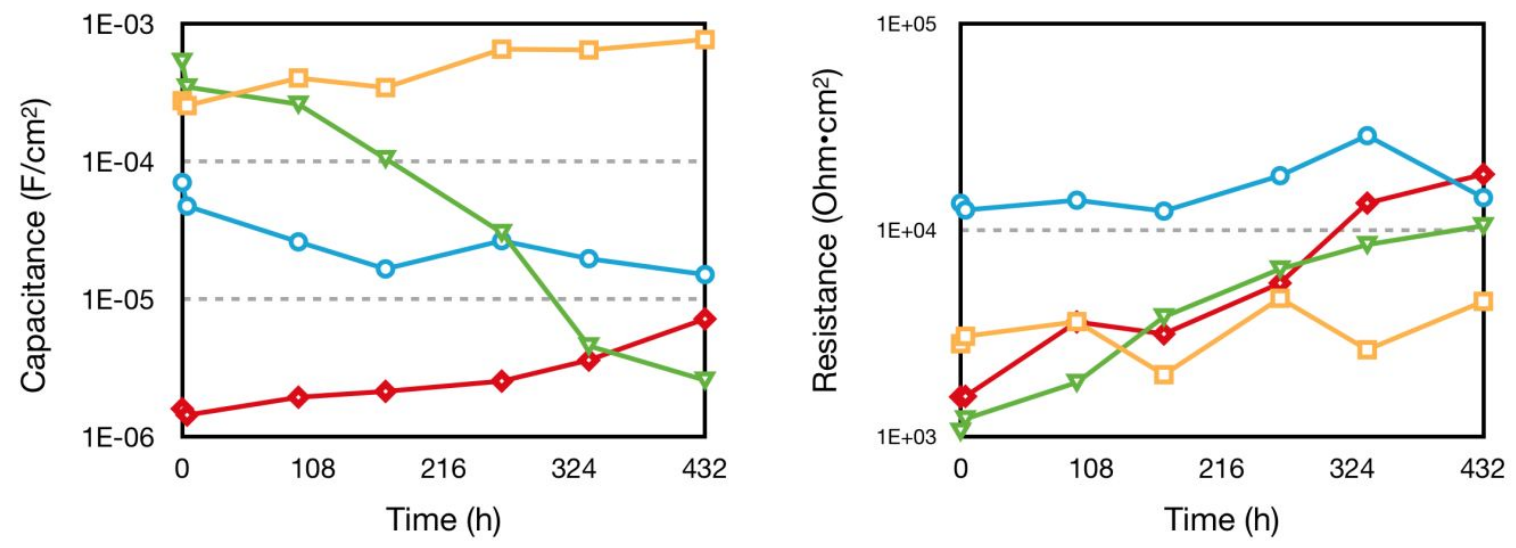

AA2024-T3 $\quad \nabla 1045$

3003-H14 $\diamond 304-2 \mathrm{~B}$

Figure S14. Electrical capacitance and resistance of the bare steel and aluminum alloys for the base line experiments (non-scaling conditions). 